\title{
Berufspolitik BvDU
}

\section{Berufsverband der Deutschen Urologen e.V.}

Urologe 2021 · 60:671-681

https://doi.org/10.1007/s00120-021-01540-6

(c) Springer Medizin Verlag GmbH, ein Teil von Springer Nature 2021
Redaktion

Dr. R. Zielke (V.i.S.d.P.)

BvDU-Geschäftsführer

Kantstr. 149

10623 Berlin

\section{Inhalt}

671 Hängepartie in der Hygiene

672 Urologen unterstützen Corona-Impfkampagne: Auch Fachärzte können ihre Patienten impfen

673 TSVG in Corona-Zeiten

674 Darmkrebs-Früherkennung trotz Pandemie: Stuhltest beim Urologen kann Leben retten

676 BvDU-Köpfe im Porträt:„SCharfe Kritik muss geäußert werden, wenn unser Gesundheitssystem nicht den Bach runter gehen soll."

677 Apropos Frauenquote - in der ambulanten Gesundheitsversorgung nicht nötig

678 Fortbildungs-Klassiker im Mai: 12. Urologisches Sommerforum in Hamburg

678 Ihr Gutes Recht: Urlaubszeit - Ausfliegen geht (dieses Jahr vielleicht), abtauchen nicht

\section{EDITORIAL}

\section{Hängepartie in der Hygiene}

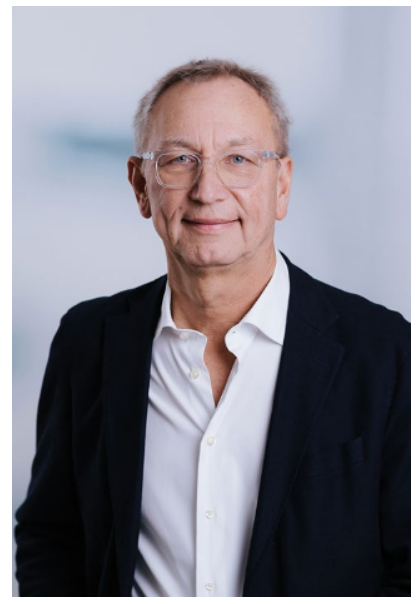

Nach nun fast zwei Jahren Hygiene-Kampagne bleiben die Fronten zum Thema Hygienekosten-Vergütung weiter verhärtet: Ende März konnte in der Sitzung des Erweiterten Bewertungsausschusses (E-BA) kein Ergebnis in Sachen Hygienekostenvergütung erzielt werden. Das Angebot, auf der einen Seite 90 Millionen Euro für Hygieneanforderungen bereitzustellen, auf der anderen Seite aber die Praxen mit 0 Euro für die Folgen der Digitalisierung im Regen stehen zu lassen, ist untragbar. Qualifizierte Medizinproduktaufbereitung und Qualitätssicherung von beispielsweise Endoskopen, Endorektalsonden und Biopsiegeräten sind primär nicht das Problem in der ambulanten Versorgung - nur dann muss auch das Geld der Leistung folgen. Wenn Gespräche und Verhandlungen scheitern, werden wir als Berufsverband andere Maßnahmen ergreifen müssen, um eine Gegenfinanzierung der unter Corona explodierenden Hygienekosten zu erwirken.

Für die Hygiene mussten schon vor Corona Praxen drauflegen. Laut einer Umfrage des Zentralinstituts für die kassenärztliche Versorgung von 2018 betragen sie durchschnittlich 24.287 Euro. Mit dem vorgeschlagenen Kompromiss würde es im Schnitt umgerechnet 900 Euro für die 100.000 Praxen in Deutschland geben. Das ist die vermeintliche Wertschätzung der Krankenkassen dafür, dass die Hausärzte und Fachärzte aktuell gerade hohen Hygienestandards erfüllen, zum Schutz ihrer Patienten genauso wie für die Praxisteams und sich selbst.

Somit halten wir als BvDU die Entscheidung des unparteiischen GBA-Mitglieds Prof. Wasem für alles andere als angemessen. Sie ist geradezu tendenziös und unterstützt die Blockadehaltung der Krankenkassen. Insofern blieb auch der KBV nichts anderes übrig, formal die Gespräche gleich abzubrechen und sich nicht auf dieser Linie abspeisen zu lassen. Als Institution des öffentlichen Rechts kann und muss sie aber nun weiterverhandeln, das heißt der E-BA muss neu liefern.

In Corona-Zeiten sollte sowohl Politik als auch Krankenkassen klar sein, dass die Hygiene vorrangig behandelt werden muss - gerade im Hinblick darauf, dass Arztpraxen massenweise gegen COVID-19 impfen. Es ist absolut unverständlich, warum die Hinhaltetaktik der Krankenkassen in der Hygiene-Thematik von der Politik und den Standesvertretern toleriert wird. Wir als Berufsverband akzeptieren das auf jeden Fall nicht und haben eine konzertierte Aktion der Verbände gestartet, um hier mit Nachdruck eine Lösung zu erzielen. Verhandeln alleine reicht nicht mehr.

Mit herzlichen Grüßen

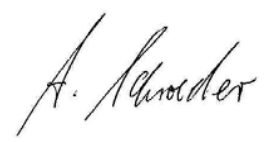

Ihr Dr. Axel Schroeder

Präsident Berufsverband der Deutschen Urologen e.V. 


\section{Urologen unterstützen Corona- Impfkampagne: Auch Fachärzte können ihre Patienten impfen}

Der Berufsverband der Deutschen Urologen (BvDU) e.V. forderte mehr Pragmatismus nach dem Impfgipfel Ende März: „Wir Fachärzte nehmen die Kanzlerin Merkel beim Wort: 20 Mio. Impfungen pro Woche sind möglich, wenn wir mit den Hausärzten gemeinsam handeln“, kommentiert BvDU-Präsident Dr. Axel Schroeder die Impf-Beratungen.,In einem ersten Schritt sollten wir Urologen unsere onkologischen Patienten gegen Corona impfen."

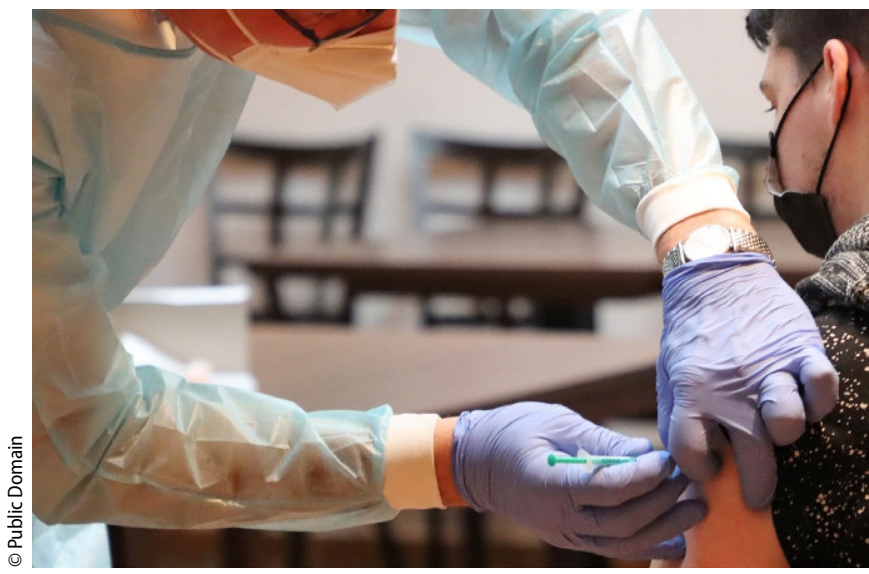

Da die Impfstoffmenge im April noch knapp sein wird, werden zunächst besonders gefährdete Personen (Priorisierung 2) entsprechend der Empfehlungen der Ständigen Impfkommission zur Impfreihenfolge geimpft. „Arztpraxen - dazu gehören auch niedergelassene Fachärzte - müssen schnellstmöglich in das Impfgeschehen einbezogen werden, um insbesondere chronische Patienten besser erreichen $\mathrm{zu}$ können“, erklärt Schroeder. „Wenn Fachärzte mithelfen sollen, muss frühzeitig mehr Flexibilität in den Impfprozess gebracht werden. Wir Urologen haben die nötige Impfkompetenz und stehen auf jeden Fall in den Startlöchern."

\section{Informationen zur COVID-19-Schutzimpfung für Urologen}

Bereits Anfang Januar hatte der Berufsverband gemeinsam mit der Deutschen Gesellschaft für Urologie e. V. (DGU) die Handreichung „Informationsmaterialien und Empfehlungen zur COVID-19-Schutzimpfung für den urologischen Alltag in Klinik und Praxis" herausgegeben. Sie soll die tägliche Arbeit von Urologen unterstützen. Neben einer Zusammenfassung der umfassenden „Mitteilung der Ständigen Impfkommission am Robert Koch-Institut für die Empfehlung der COVID-19-Impfung und die dazugehörige wissenschaftliche Begründung (STIKO-Empfehlung zur COVID-19-Impfung) findet sich darin ein Extrakt aus der „Verordnung des Bundesgesundheitsministeriums zum Anspruch auf Schutzimpfung gegen das Coronavirus SARS-CoV-2 (Coronavirus-Impfverordnung) vom 18. Dezember 2020. Dritter Teil dieser Schrift ist eine Empfehlung zur mRNA-basierten Impfung gegen COVID19 für uro-onkologische Patienten unter medikamentöser Tumortherapie, basierend auf den aktuellen STIKO-Veröffentlichungen „Impfen bei Immundefizienz (Teil III und IV)“.

\section{Impfen in der Praxis, jede Praxis zählt}

Der AstraZeneca-Impfstoff ist wieder zugelassen. Mit der Zulassung weiterer Impfstoffe, z. B. Johnson \& Johnson seit dem 11.03.2021, könnte die Verfügbarkeit von Impfstoffen in Deutschland schon bald angehoben werden. Sei es in den Impfzentren als auch in den Arztpraxen. Nach Ostern sollen Haus- und Fachärzte mit ihren Praxen, wohnortnah und flächendeckend jetzt Schritt für Schritt eingebunden werden und gegen Corona impfen können. Dabei gilt es die aktuellen Empfehlungen der STIKO zu beachten, die Impfstoffwahl als auch Alter und Begleiterkrankungen, inklusive Nebenwirkungen zu berücksichtigen. Das Nebenwirkungsspektrum wurde jetzt neu bewertet und angepasst. Bis auf weiteres ist keine individuelle Wahl eines spezifischen Impfstoffes möglich. Das kann sich mit zunehmender Verfügbarkeit ändern. Auch kann neu priorisiert werden. Dann muss gelten: Wer will, der darf!

Praxen, die impfen möchten, sollten die regionalen Impfbestimmungen ihrer jeweiligen Landesbehörden und Kassenärztlichen Vereinigen beachten. Außerdem bieten viele Praxisverwaltungssysteme mittlerweile Software zum Impfmanagement (teilweise lizenzkostenfrei) an.

\section{COVID-19 Impf-App für Ärzte und medizinisches Fachpersonal}

Als Teil einer nationalen COVID-19 Impfstrategie empfiehlt der BvDU die Begleitung Geimpfter per DIFA1App. Mit dieser neuen kostenlosen Smartphone-App können Ärzte und ihre Mitarbeitende, die zu den priorisierten Empfängern der neuen Corona-Impfstoffe gehören, mögliche Begleiterscheinungen und vor allem auch die Effektivität im Schutz vor Erkrankung - gerade auch vor dem Hintergrund der aufgetretenen Virusmutationen - einfach dokumentieren. Sie wird vom DIFA (Deutsches Institut für Fachärztliche Versorgungsforschung) zur Verfügung gestellt:

\section{Fünf Gründe für die DIFA1 App}

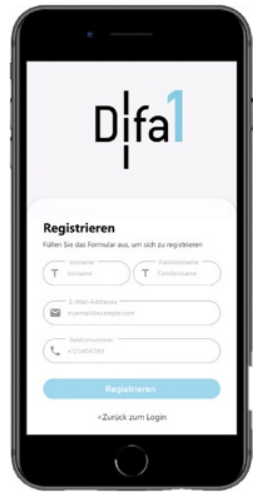

1. Gesundheitsfachpersonal schafft Vertrauen

2. Optimierung der Datenbasis hinsichtlich der Impfstoff-Effektivität

3. Datenerfassungsmöglichkeit unabhängig vom Impfdatum

4. Sichere Nutzung dank Zweifaktor-Authentifizierung

5. Verifizierungsfähigkeit im Fall von Impfdurchbrüchen

\section{Mit Sicherheit Impfen! Das WIR zählt!}

Umfangreiches Informationspaket der KBV für Praxen

Weitere Informationen zur COVID-19-Schutzimpfung in Arztpraxen finden Sie zudem auf der Internetseite der KBV. Die Informationen dort werden fortlaufend aktualisiert.

BvDU-Statement, 25.03.2021 


\section{TSVG in Corona-Zeiten}

In Corona-Zeiten überschlagen sich die täglichen Meldungen und Veränderungen. Es gilt aber trotzdem, auch andere Themen im Blick zu behalten. Zum Beispiel die Auswirkungen des Terminservice- und Versorgungsgesetzes (TSVG) unter Corona-Bedingungen.

Mit Inkrafttreten des TSVG am 11.05.2019 sollten für Patienten einige Verbesserungen bei der Inanspruchnahme gesundheitlicher Leistungen geschaffen werden durch Einrichtung von Terminservicestellen, einer bundeseinheitlichen Patienten-Servicenummer 116 117, der Überarbeitung der Heilmittelrichtlinie 1/21, durch die geplante Einführung einer elektronischen Patientenakte sowie ab dem 01.10.2021 dann die Einführung der elektronischen Arbeitsunfähigkeitsbescheinigung (eAU).

Sogenannte TSVG-Fälle (TSS-Vermittlungsfall, TSS-Akutfall, HAVermittlungsfall, offene Sprechstunde und Neupatienten) werden extrabudgetär vergütet, führen aber zu einer Bereinigung des MGV (morbiditätsbedingten Gesamtvergütung). Diese wurde zunächst für $1 \mathrm{Jahr}$ beschlossen und endete mit dem 31.08.2020.

Das TSVG sieht vor, dass die MGV basiswirksam um den Wert der bislang zulasten der MGV abgerechneten förderbaren Leistungen zu verringern ist, wobei diese Leistungen unter Berücksichtigung der bisherigen Auszahlungsquoten zu bewerten sind. Bei dieser sogenannten Bereinigung dürfen gemäß TSVG jedoch nur die jeweils im ersten Jahr nach Inkrafttreten von den Leistungserbringenden entsprechend gekennzeichneten Leistungen berücksichtigt werden. Die Begrenzung auf ein Jahr sollte vermutlich sicherstellen, dass zukünftig erwartete

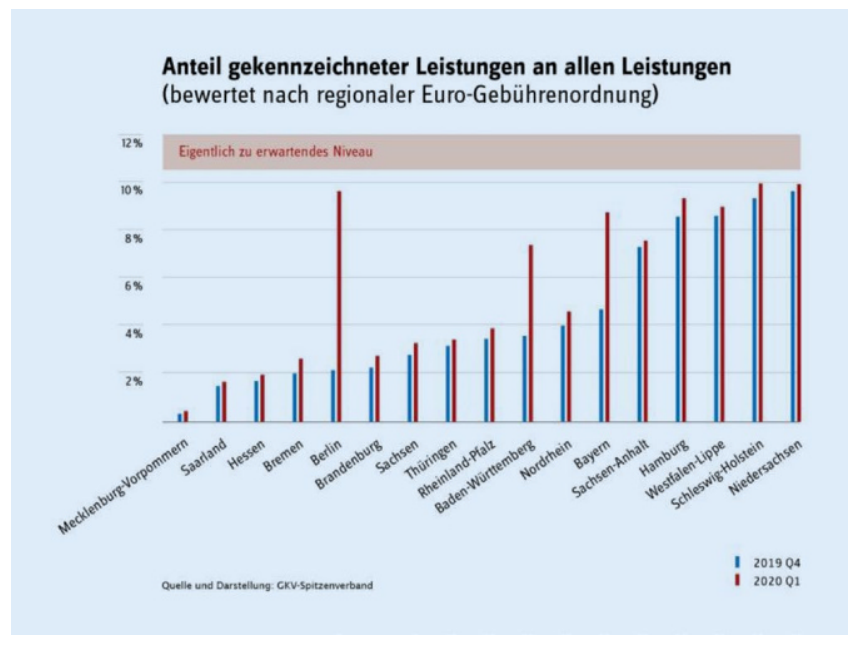

Mengensteigerungen in vollem Umfang zusätzlich vergütet werden und nicht mehr die MGV schmälern.

In der Corona-Pandemie kam es aber nicht zum (wie durch den Gesetzgeber) erwarteten kompensatorischen Anstieg der Einnahmen im EGV-Bereich aus der offenen Sprechstunde und durch Neupatienten. Im Gegenteil!

Hierzu zählt neben dem allgemeinen Inanspruchnahmerückgang auch der Vorrang der Kennzeichnung und Vergütung von Leistungen bei klinischem Verdacht auf eine Infektion oder bei einer nachgewiesenen Infektion mit dem Coronavirus SARS-CoV-2 als sogenannter

\section{Stellen- und Hospitationsbörse \\ Eine Initiative der GeSRU unterstützt durch den BvDU}
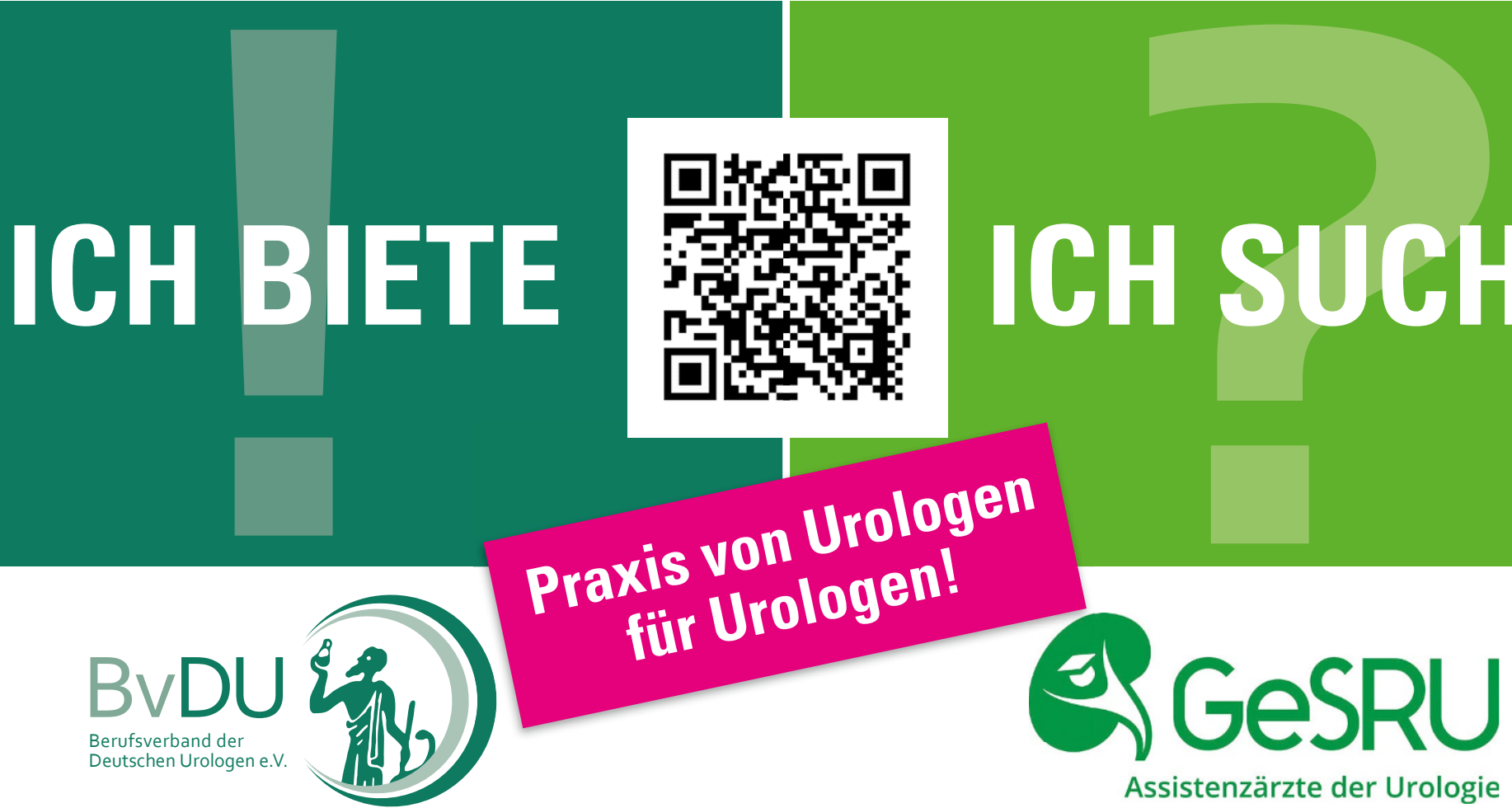
nicht vorhersehbarer Anstieg des morbiditätsbedingten Behandlungsbedarfs gemäß \$87a Abs. 3 Satz 4 SGB V.

In den einzelnen KV-Bereichen war in den letzten Quartalen eine sehr unterschiedliche Entwicklung der TSVG-Zahlen und der damit verbundenen Bereinigung zu verzeichnen.

\section{Kommentar}

Mit dem TSVG ist neben einer zügigen Inanspruchnahme gesundheitlicher Leistungen bei den TSVG-Fällen eine extrabudgetäre Vergütung von der ärztlichen Selbstverwaltung verankert worden. Ein Schritt in Richtung Entbudgetierung.

Als Berufsverband begrüßen wir diese Ausbudgetierung einzelner Leistungen (hier: offene Sprechstunde, neuer Patient, TSS-Akutfall etc.). Diese waren bisher EBM-Leistungen, die unter den Budgetdeckel gefallen waren und somit nicht voll bezahlt wurden. Langfristiges Ziel bleibt für uns als BvDU die konsequente Entbudgetierung aller ärztl. Leistungen. Damit dieser Ansatz von Anfang an nicht durch die Krankenkassen unterlaufen wird, galt es das Bereinigungsverfahren nach dem 30.08.2020 zu beenden, welches von den Krankenkassen in der Corona-Pandemie abgelehnt wurde. Der Verband der Ortskrankenkassen ging davon aus, dass diese Bereinigung u.a. wegen geringere Leistungsmenge in der Corona-Phase unvollständig erfolgt ist.

Der Spitzenverband der Fachärzte e. V. konnte sich für die grundversorgenden Fachärzte im Gesetzgebungsverfahren (Weitentwicklungsgesetz der Gesundheitsversorgung) durchsetzen, ein zusätzliches Korrekturverfahren in der Bereinigung dieser zu streichen. Damit bleibt es bei einer einmaligen Bereinigung bis zum 30.08.2020. Eine Zunahme der TSVG-Fälle, insbesondere aktuell in der Pandemie (In-

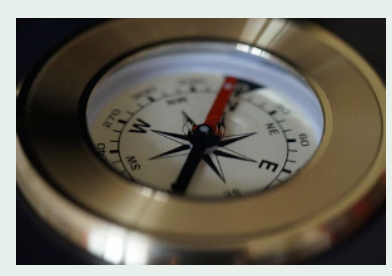

\section{Kommentar}

Mit dem TSVG ist neben einer zügigen Inanspruchnahme gesundheitlicher Leistungen bei den TSVG-Fällen eine extrabudgetäre Vergütung von der ärztlichen Selbstverwaltung verankert worden. Ein Schritt in Richtung Entbudgetierung.

Als Berufsverband begrüßen wir diese Ausbudgetierung einzelner Leistungen (hier: offene Sprechstunde, neuer Patient, TSS-Akutfall etc.). Diese waren bisher EBM-Leistungen, die unter den Budgetdeckel gefallen waren und somit nicht voll bezahlt wurden. Langfristiges Ziel bleibt für uns als BvDU die konsequente Entbudgetierung aller ärztl. Leistungen. Damit dieser Ansatz von Anfang an nicht durch die Krankenkassen unterlaufen wird, galt es das Bereinigungsverfahren nach dem 30.08.2020 zu beenden, welches von den Krankenkassen in der Corona-Pandemie abgelehnt wurde. Der Verband der Ortskrankenkassen ging davon aus, dass diese Bereinigung u. a. wegen geringere Leistungsmenge in der Corona-Phase unvollständig erfolgt ist Der Spitzenverband der Fachärzte e.V. konnte sich für die grundversorgenden Fachärzte im Gesetzgebungsverfahren (Weitentwicklungsgesetz der Gesundheitsversorgung) durchsetzen, ein zusätzliches Korrekturverfahren in der Bereinigung dieser zu streichen. Damit bleibt es bei einer einmaligen Bereinigung bis zum 30.08.2020. Eine Zunahme der TSVG-Fälle, insbesondere aktuell in der Pandemie (Infektsprechstunde), geht jetzt zu Lasten der Krankenkassen. Dies unterstreicht das Potenzial der fortgesetzten Entbudgetierung. fektsprechstunde), geht jetzt zu Lasten der Krankenkassen. Dies unterstreicht das Potenzial der fortgesetzten Entbudgetierung.

Das veranlasste die Bundesregierung zu einem Entwurf innerhalb des Gesetzes zur Weiterentwicklung der Gesundheitsversorgung (kurz: GVWG), Bearbeitungsstand 19.02.2021.

Im Fokus liegen vor allem KVen, die geringe TSVG-Fallzahlen haben. Hier soll $\$ 87$ a Abs. 3 regeln, wie nach Vorgaben des EBA die KVen ab dem 01.10.2021 bis zum 30.06.2022 vierteljährlich ein Verfahren zur Korrektur der Bereinigung der MGV durchzuführen haben.

Dazu bezieht die KBV wie folgt Stellung:

Es müssen keine Korrekturen vorgenommen werden, wenn die Daten den zu erwartenden Leistungsmengen entsprechen oder wenn die Kassenärztliche Vereinigung die Abrechnungsdaten bereits korrigiert hat.

Wenn durch die Corona-Pandemie ein Rückgang der Leistungsmengen der offenen Sprechstunde erfolgt ist, sollte als Korrektur bzgl. der offenen Sprechstunde eine Hochrechnung mit nicht von der Pandemie betroffener Quartale erfolgen, um die Corona-Effekte zu kompensieren. Weitere Korrekturen sind dann nicht erforderlich.

Für die weiteren Bereinigungstatbestände nach Satz 5 liegen keine Anhaltspunkte vor, dass aufgrund des Abrechnungsverhaltens der Vertragsärzte oder wegen der Corona-Pandemie eine Unterbereinigung vorliegen könnte. Eine erneute Korrektur würde hier zu einer Doppelbereinigung führen. Regionale Spezifitäten können zudem auf Bundesebene nicht erfasst werden.

Vor diesem Hintergrund sollen die Gesamtvertragspartner auf Landesebene prüfen, ob eine Korrektur in diesem KV-Bezirk notwendig ist und in diesem Fall mit Wirkung ab dem 1. Oktober 2021 ein entsprechendes Korrekturverfahren vereinbaren. Sofern bereits eine Korrektur durch die KVen stattgefunden hat, ist kein weiteres Korrekturverfahren vorzusehen.

Autorin: Catrin Steiniger, 1. Vizepräsidentin BvDU (komm.) und BvDU-Landesvorsitzende Brandenburg, 07.03.2021

\section{Darmkrebs-Früherkennung trotz Pandemie: Stuhltest beim Urologen kann Leben retten}

Früherkennung tut nicht weh. Werden Krebserkrankungen frühzeitig erkannt und behandelt, bestehen oftmals gute bis sehr gute Heilungsaussichten. Dennoch gehen nur $40 \%$ der Männer regelmäßig zur Krebsvorsorge. Corona bedingt ist diese Zahl laut der Weltgesundheitsorganisation noch weiter gesunken.

„Im Rahmen der Früherkennungs-Untersuchung bieten wir Urologen unseren Patienten zur Darmkrebs-Vorsorge einen immunologischen Stuhltest an und weisen auf die präventive Dickdarm-Spiegelung hin“, erklärt Dr. Axel Schroeder, Präsident des Berufsverbands der Deutschen Urologen e. V. „Es ist wichtig, unsere Patienten dazu ermutigen, auch während der Pandemie wichtige Vorsorgeuntersuchungen wahrzunehmen." Eine Studie der University of Exeter zeigte unlängst, dass bei Patienten mit niedrigem Risiko für Darmkrebs immunologische Stuhltests ein gutes Mittel seien, um die weitere Diagnostik zu 


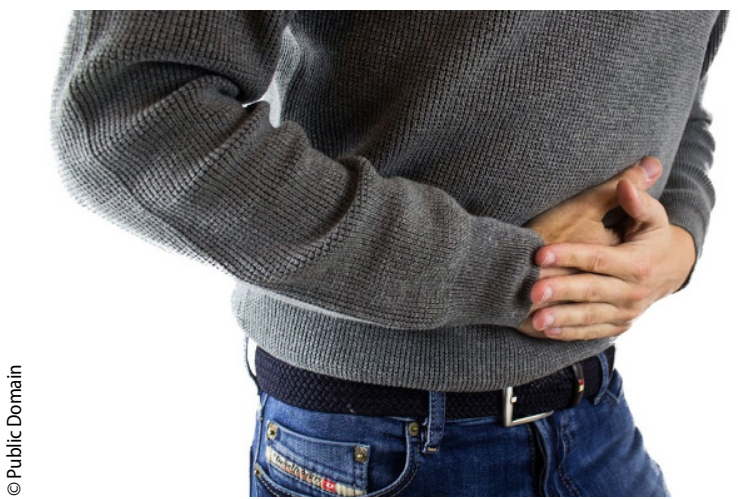

steuern. „Verzögerungen bei Therapien wie Operationen, Bestrahlung oder Chemotherapie haben erhebliche Auswirkungen auf die Sterblichkeit von Krebspatienten - das sind weitere fatale Folgen von Corona", so Schroeder weiter.

\section{Kosten für Stuhltest übernehmen Krankenkassen}

Darmkrebs gehört zu den drei häufigsten Krebserkrankungen bei Männern sowie Frauen in Deutschland und tritt überwiegend $a b$ dem 50. Lebensjahr auf. „Im Darmkrebs-Monat März möchten wir als Berufsverband noch einmal verstärkt darauf hinweisen, dass der immunologische Stuhltest beim Urologen Leben retten kann", betont Schroeder. „Bei vielen Patienten wird so Darmkrebs entdeckt, der ansonsten wohl längere Zeit verborgen geblieben wäre. “ Die Kos- ten für den Test werden von den gesetzlichen Krankenkassen im Alter von 50-54 Jahren einmal übernommen, wenn keine Früherkennungsdarmspiegelung (Koloskopie) in Anspruch genommen wurde. Darüber hinaus bieten einige Krankenkassen das Früherkennungsprogramm je nach individuellem Risikoprofil des Patienten, wie beispielsweise familiärer Vorbelastung, bereits vor der angegebenen Altersgrenze oder in kürzeren Intervallen an. Bei positivem Befund des immunologischen Stuhltests wird eine Darmspiegelung beim Gastroenterologen durchgeführt, mittels derer eine Diagnose gestellt wird. „Die Heilungschancen von Darmkrebs bei frühzeitiger Entdeckung liegen bei über $90 \%$ “, so Schroeder. „Früherkennung hilft heilen - das kann man den Patienten gar nicht oft genug sagen."

BvDU-Pressemitteilung, 16.03.2021

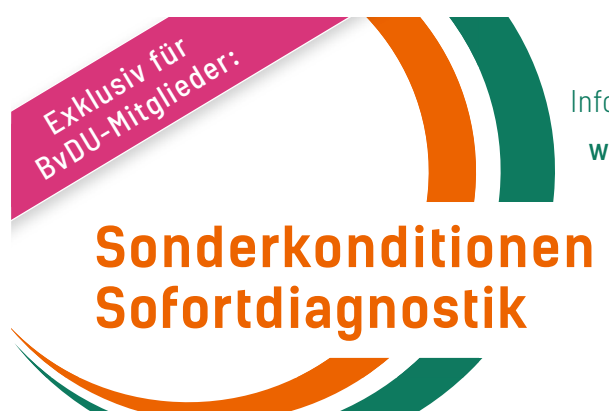

Weitere Informationen unter: www.sgdu-mbh.de

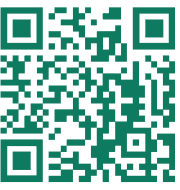

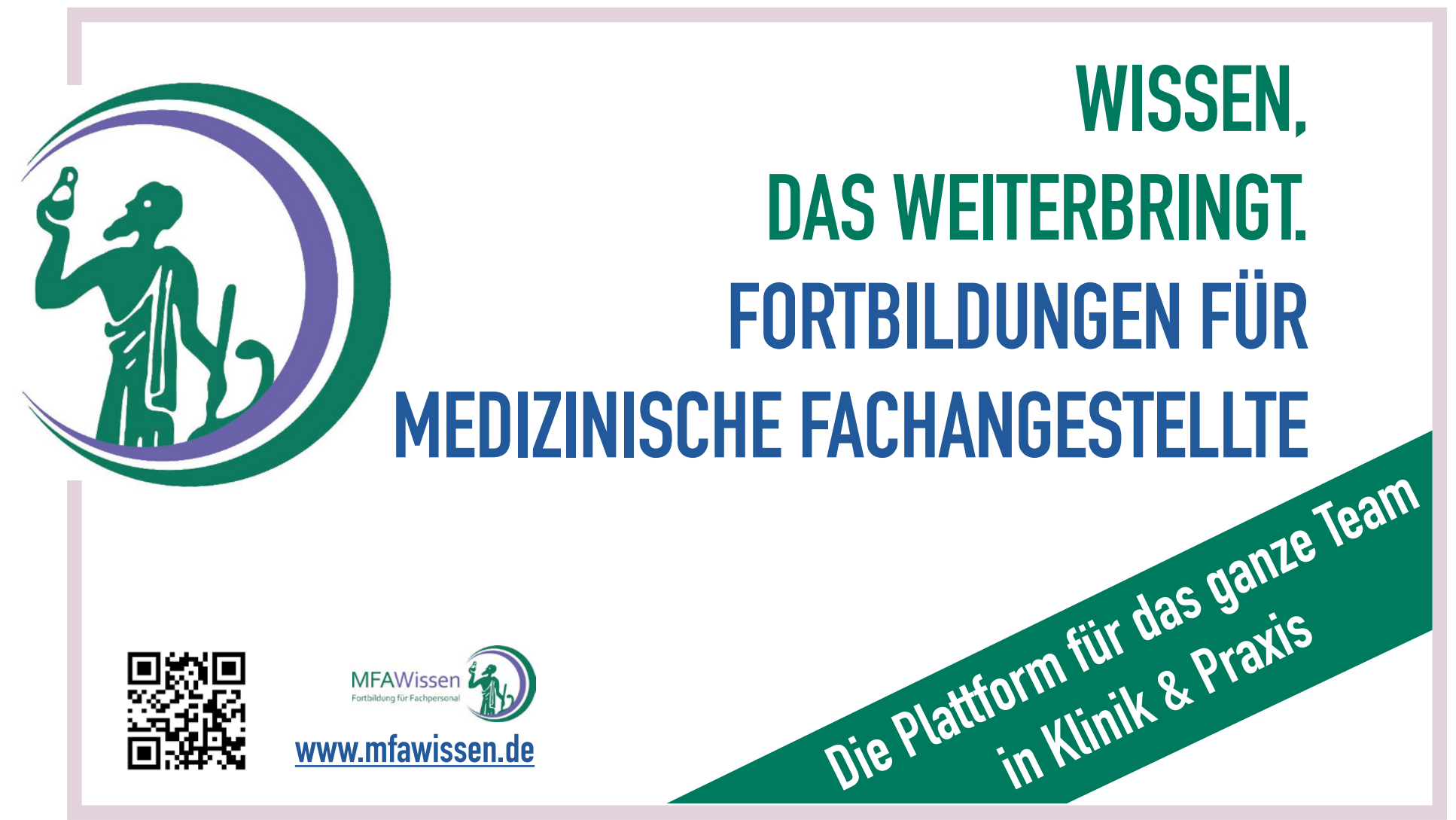




\section{BvDU-Köpfe im Porträt: „,Scharfe Kritik muss geäußert werden, wenn unser Gesundheitssystem nicht den Bach runter gehen soll."}

Seit Ende 2020 ist Dr. Peter Kollenbach, Landesvorsitzender Hessen und Leiter des Sachausschusses Qualitätsmanagement des BvDU, neuer Sprecher des Hauptausschusses. Im Interview spricht er über seine berufspolitischen Ziele und darüber wie er sich für mehr Geschlossenheit innerhalb des Berufsverbands einsetzt.

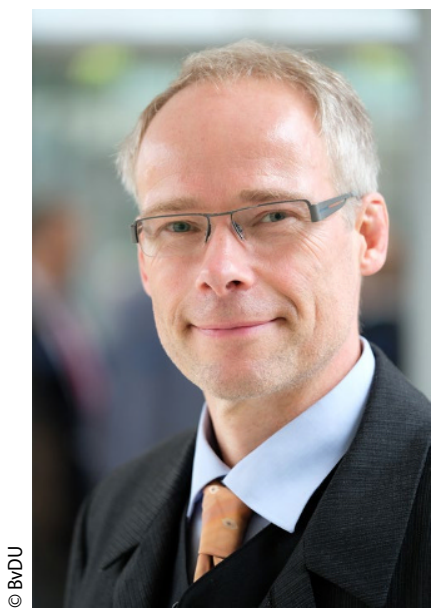

1. Herr Dr. Kollenbach, bitte stellen Sie sich kurz vor.

PK: Mein Name ist Dr. Peter Kollenbach und ich arbeite als Facharzt für Urologie in einer Gemeinschaftspraxis in Kassel mit insgesamt fünf weiteren Kollegen. Darüber hinaus bin ich auch als Belegarzt tätig.

2. Seit wann sind Sie BvDU-Mitglied und warum sind Sie damals beigetreten?

PK: Berufspolitisch aktiv wurde ich erstmals im Jahr 2009 - und zwar als eines der Gründungsmitglieder der Genossenschaft Hessische Urologen eG (HUeG). Diese regionale Interessensvertretung hat das Ziel, niedergelassene Urologen in Hessen zu stärken, damit die Existenz der Praxen und somit die ambulante Betreuung von Patienten gewährleistet wird. Später war ich Aufsichtsratsmitglied, Vorstand und zuletzt Vorstandssprecher der HUeG. Der Eintritt in den BvDU im Jahr 2013 war für mich dann eine konsequente Fortführung meines berufspolitischen Engagements über die Bundeslandgrenzen hinaus. Von Anfang an hatte ich das Amt des Landesvorsitzenden von Hessen inne. Als engagiertes Mitglied im BvDU-Hauptausschuss wurde ich Ende letzten Jahres dann zusätzlich als dessen Sprecher gewählt.

\section{Was hat Sie dazu bewegt, in der Berufspolitik aktiv zu werden?}

PK: Ich wollte gegen die Ohnmacht gegenüber den Akteuren im Gesundheitssystem kämpfen. In meinen Augen agieren diese teils willkürlich und verfolgen nur eigene Interessen. Außerdem war mein Anliegen, mich für mehr Transparenz im Abrechnungssystem einzusetzen. Denn ohne eine Lobby stehen diejenigen, die einfach nur gute Medizin machen möchten, in diesem System auf verlorenem Posten.

4. Wie kam es zu dem Entschluss, neben Ihren ganzen anderen Ämtern, zusätzlich noch die Funktion des Hauptausschuss-Sprechers anzunehmen?

PK: Jeder Landesverband ist der verlängerte Arm des BvDU in die jeweiligen Regionen mit den unterschiedlichen KVen und Landesärztekammern hinein. Über diese Arbeit tauschen sich die Landesvorsitzenden im Rahmen der Hauptausschusssitzungen regelmäßig aus. Aber nicht alles läuft dabei immer so richtig rund. Ich möchte daran mitarbeiten, dass die Abstimmung untereinander künftig besser funktioniert. Die breite Akzeptanz unter den übrigen HauptausschussMitgliedern hat mich bestärkt, der Richtige für diesen Posten zu sein.
5. Was reizt Sie an Ihrer Aufgabe besonders und mit welchen Herausforderungen sehen Sie sich konfrontiert?

PK: Ich möchte Dinge bewegen und nicht einfach wie ein Schaf hinterherlaufen. Verantwortung zu übernehmen ist wichtig. Das gilt für jeden Urologen in seinem eigenen ganz persönlichen Umfeld, aber auch der Blick über den Tellerrand ist wichtig und notwendig. Die Arbeit im Hauptausschuss hat meinen Horizont deutlich erweitert. Die Verflechtungen bei dem zunehmend intransparenten Gesundheitssystem sind für jemanden, der sich nicht damit beschäftigt, nicht zu durchschauen. Ich gewinne laufend neue Erkenntnisse hinzu, trotzdem bleiben einige Siegel verschlossen. Den richtigen Hebel, den richtigen Ansatzpunkt zu finden, ist manchmal gar nicht so einfach. Das fängt bei den Mitgliedern an, welche motiviert werden müssen aktiv mit zu denken und zu arbeiten. Der richtige Stil im Umgang mit den Krankenkassen, der Politik ist nicht immer leicht zu finden. Ich bin der Meinung, dass scharfe Kritik geäußert werden muss, wenn unser Gesundheitssystem nicht den Bach runter gehen soll.

\section{Was kritisieren Sie an der derzeitigen Gesundheitspolitik?}

PK: Ich sehe die derzeitigen Entwicklungen mit Sorge. Das deutsche Gesundheitssystem erscheint zunehmend kopflos und wird den Partikularinteressen von Politik und Krankenkassen geopfert. Hier muss klare Kante gezeigt werden, die Leistungserbringer müssen laut auftreten und die Honorierung ihrer Leistung einfordern. Die ärztliche Selbstverwaltung in der derzeitigen Form ist ein Hohn, Vertragsverhandlungen mit den Krankenkassen werden schlecht geführt. Mit dem Ergebnis, dass es immer mehr Mangelverwaltung gibt, die dann in zunehmender Sanktionierung mündet. Vielen niedergelassenen Kollegen bleibt oft nichts anders übrig, als das Handtuch zu schmeißen. Dies hat die Politik noch nicht erkannt. Gesundheitsökonomen sind mittlerweile wichtiger geworden, als die Leistungserbringen. Das muss sich in naher Zukunft ändern.

\section{Was bedeutet das konkret für die Urologie?}

PK: In der Urologie sind insbesondere die Niedergelassenen gefährdet. Die schlechten Verhandlungen der Kassenärztlichen Vereinigungen mit der nachfolgenden Mangelverwaltung führen zu immer weiteren Einschnitten und Restriktionen. Aufgaben, die die Urologen gerne übernehmen würden, werden an andere Institutionen übertragen, da deren Vergütung hierfür besser ist oder diese ihre Ausgaben querfinanzieren können. Diese Institutionen werden dann in der Folge hofiert, die Gelder von den niedergelassenen Urologen abgezweigt und weitere Restriktionen und Einschnitte werden folgen.

\section{Als Belegarzt tragen Sie ja nicht nur die Brille des ambulanten Be-} reichs - wie sehen Sie die derzeitige sektorübergreifende Versorgung in der Urologie?

PK: Durch meine Tätigkeit als Belegarzt agiere ich als Bindeglied zwischen niedergelassener und stationärer Urologie - ich bin ja qua-

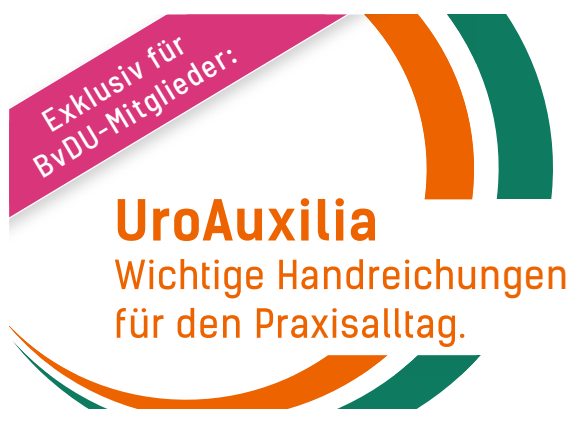
www.sgdu-mbh.de

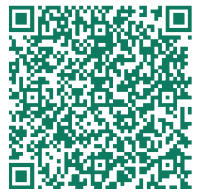




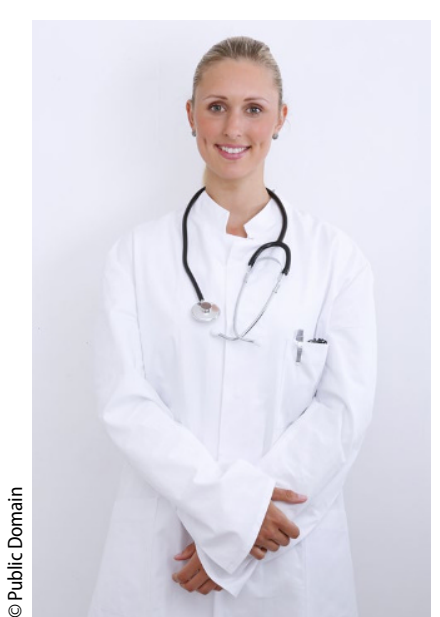

\section{Zahnärztinnen}

Unter den zahnmedizinischen Studierenden sind Frauen bereits seit Jahren in der Mehrheit. Die in Praxen angestellten Zahnärztinnen kommen inzwischen bereits auf einen Anteil von über $60 \%$. So war es nur eine Frage der Zeit, bis dieser Trend auch bei zahnärztlichen Existenzgründungen ankommen würde: 2019 haben sich mit $51 \%$ zum ersten Mal mehr Frauen als Männer niedergelassen.

\section{Apothekerinnen}

Bereits seit Jahren lassen sich deutlich mehr Apothekerinnen als Apotheker nieder. 2019 waren $59 \%$ der Existenzgründer Frauen, entsprechend nur 41 \% Männer. Ein Blick in die Regionen zeigt, dass vor allem im Süden und Osten Deutschlands Apothekerinnen stark vertreten waren. Dort belief sich ihr Anteil auf $67 \%$ beziehungsweise $61 \%$.

„Dass die künftige Gesundheitsversorgung zunehmend weiblich wird, zeichnet sich seit längerem ab, Frauen sind bei den Studierenden in allen Heilberufsfächern deutlich stärker vertreten als Männer", sagt Daniel Zehnich, Leiter des Bereichs Gesundheitsmärkte und Gesundheitspolitik der apoBank. „Doch proportional gesehen entscheiden sie sich im Vergleich zu ihren männlichen Kollegen seltener für eine Niederlassung. Für eine gut funktionierende ambulante Gesundheitsversorgung ist es daher besonders wichtig, für Rahmenbedingungen zu sorgen, die den Lebensentwürfen von Frauen besser entsprechen. Denn unsere Studien zeigen, dass Heilberuflerinnen häufig das Gefühl haben, sich zwischen Kind und Karriere entscheiden zu müssen. Benötigt werden also innovative, flexible Formen der Berufsausübung als eigene Chefin oder kooperative Praxisformen, die es ermöglichen, die individuellen Vorstellungen vom eigenen Arbeitspensum zu realisieren."

Quelle: Pressemitteilung apoBank, 08.03.2021

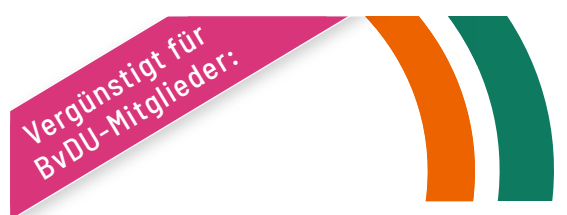

Fortbildungsveranstaltungen

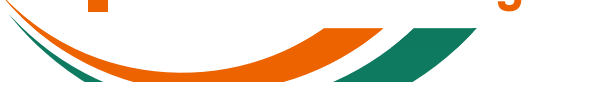

Fortbildungs-Klassiker im Mai: 12. Urologisches Sommerforum in Hamburg

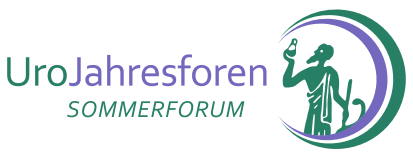

Bereits zum zwölften Mal in Folge wird das Urologische Sommerforum stattfinden. Derzeit als Präsenzveranstaltung vom 28. bis 29. Mai in Hamburg geplant, hält das Programm nicht nur aktuelle Themen des Versorgungsalltages in der Urologie wie Informationen zur aktuellen Leitlinie Harnwegsinfekt und zum Prostata-, Blasen- und Nierenzellkarzinom bereit, sondern liefert auch Wissenswertes rund um die SARS CoV2-Impfstoffen und die HPV-Impfung. Darüber hinaus werden die Teilnehmer mehr über Innovationen und Zukunftstechnologien in der Urologie erfahren.

Wie schon in den vergangenen Jahren, wird es für Medizinische Fachangestellte am ersten Veranstaltungstag Fortbildungsangebote geben. Neben spannenden Vorträgen bieten die UroJahresforen immer auch vielfältige Möglichkeiten zum kollegialen Austausch. Sollten die pandemiebedingten Bestimmungen allerdings nur eine Hybrid- oder eine ausschließliche Online-Veranstaltung zulassen, werden wir Sie hierüber rechtzeitig informieren.

Weitere Informationen und Anmeldung unter www.sgdumbh.de

\section{Ihr Gutes Recht: Urlaubszeit - Ausfliegen geht (dieses Jahr vielleicht), abtauchen nicht}

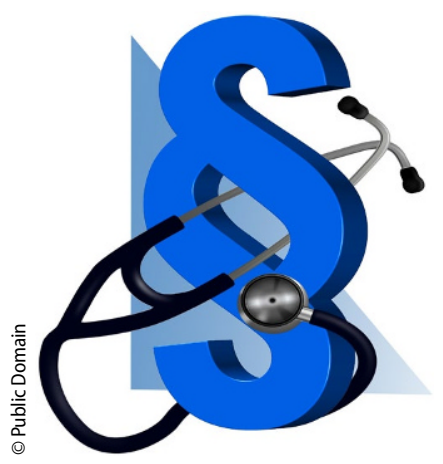

Auch wenn sich die Urlaubspläne in diesem Jahr bei vielen anders gestalten als sonst, bleiben die zu treffenden Vorkehrungen die gleichen. Da bei Vertragsärzten auch während der Urlaubszeit die medizinische Versorgung der Patienten durchgehend gewährleistet sein muss, muss eine Vertretung organisiert werden.

Grundsätzlich hat der Vertrags-

Weitere Informationen unter: www.sgdu-mbh.de

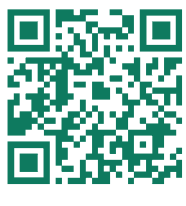

arzt seine vertragsärztliche Tätigkeit gem. $\$ 32$ Abs. 1 Ärzte-ZV persönlich in freier Praxis auszuüben. In diesem Rahmen muss er ein Mindestangebot an Sprechstunden zur Verfügung stellen und sich in Abwesenheit - sei es Urlaub, Krankheit oder die Teilnahme an einer ärztlichen Fortbildung - vertreten lassen. Die Vertretungspflicht gilt jedenfalls bei Verhinderung ab einer Woche.

Die Patienten während der Urlaubszeit einfach auf den ärztlichen Bereitschaftsdienst der KV zu verweisen, um sich die Suche nach einem Vertreter zu sparen, ist nicht erlaubt.

Das gilt im Einzelnen: 


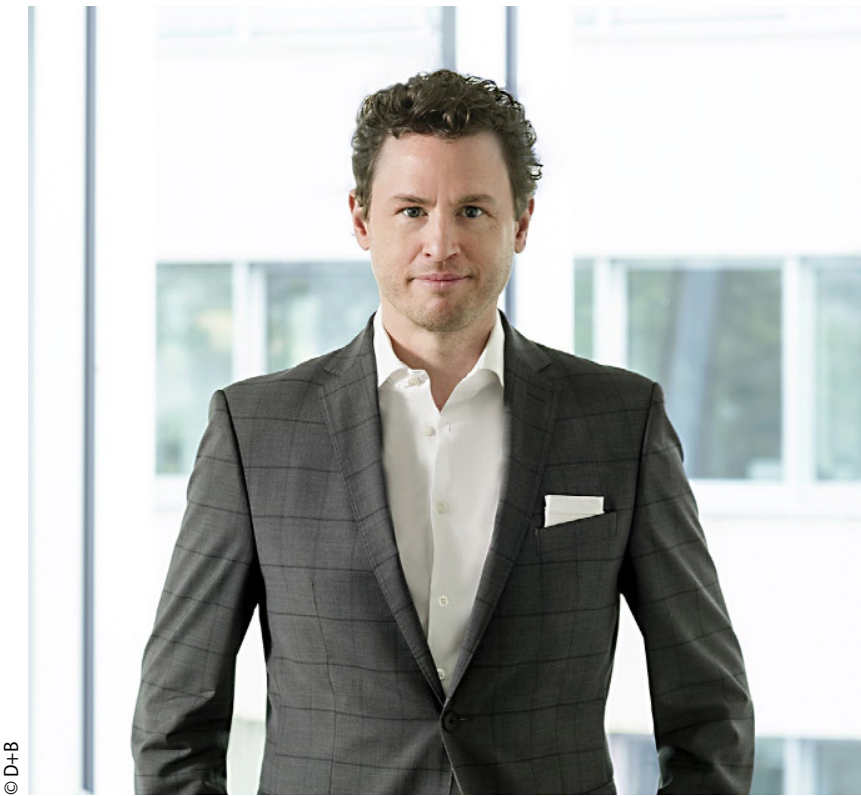

$\Delta$ Dr. Thomas Willaschek

\section{Die persönliche Vertretung}

Eine persönliche Vertretung liegt dann vor, wenn sich ein Vertragsarzt in seiner eigenen Praxis durch einen anderen, externen Arzt vertreten lässt. Dabei erbringt der Vertreter die Leistungen im Namen des zu vertretenden Arztes und unter Angabe von dessen LANR und BSNR. Der externe Vertreter erhält eine Vergütung vom Vertretenen und erlangt keinen Honoraranspruch gegenüber der KV. Bitte beachten: Im Zweifelsfall liegt ein sozialversicherungspflichtiges Arbeitsverhältnis vor, die lange Zeit übliche Honorarvertretung (freie Mitarbeit) kommt bestenfalls noch in der Einzelpraxis in Frage. Schließen Sie also einen schriftlichen Arbeitsvertrag!

\section{Die kollegiale Vertretung}

Eine weitere Möglichkeit ist die sogenannte kollegiale Vertretung. In diesem Fall wird die Praxis des zu vertretenden Arztes geschlossen, die Vertretung erfolgt durch einen Kollegen in dessen eigener Praxis. Damit das funktioniert, muss eine räumliche Nähe zwischen den Praxisstandorten vorhanden sein. Vorausschauend könnten die Patienten gebeten werden, den Vertreter nur für akute Fälle in Anspruch zu nehmen, um eine Überlastung der Vertreterpraxis zu verhindern.

\section{Keine „echte“" Vertretung}

Keine Vertretung im Sinne von $\$ 32$ Ärzte-ZV liegt hingegen vor, wenn in einem MVZ oder in einer BAG ein dort tätiger Vertragsarzt den Ausfall seines Kollegen „abfängt“, also eine interne Vertretung stattfindet. Das kann wiederum anders aussehen, wenn ein Angestellter seine vom Zulassungsausschuss genehmigte Arbeitszeit ausweitet, um die „Lücke zu füllen“. Hier kommt es auf den Einzelfall an.

\section{Anforderungen an den Vertreter}

Der Vertragsarzt darf sich gem. $\$ 32$ Abs. 1, S. 5 Ärzte-ZV grundsätzlich nur von einem Arzt vertreten lassen, der eine abgeschlossene Weiterbildung im selben Fachgebiet wie der zu vertretende Arzt hat, wenn also Fachgebietsgleichheit vorliegt. Eine vertragsärztliche Zulassung hingegen ist keine Voraussetzung für die Vertretung.

Besitzt der Vertreter die Genehmigung, qualifizierte Leistungen zu erbringen, die der zu vertretende Arzt nicht vorweisen kann, so darf auch der Vertreter diese Leistungen für den Zeitraum der Vertretung nicht abrechnen. Umgekehrt darf gem. \$14 BMV-Ä der Vertreter keine Leistungen erbringen, für die nur der zu vertretende Arzt, jedoch nicht der Vertreter selbst, über eine entsprechende Qualifikation verfügt (manche KVen verlangen, wohl zu Unrecht, auch eine Abrechnungsgenehmigung des Vertreters).

\section{Anzeige- oder Genehmigungsplicht?}

Wenn der Urlaub nicht länger als eine Woche andauert, muss die Abwesenheit der KV zwar nicht mitgeteilt werden, jedoch sollten in jedem Fall die Patienten rechtzeitig über den Urlaub informiert werden, z. B. durch einen Aushang in der Praxis, an der Tür und auf der Homepage sowie durch eine Ansage auf dem Anrufbeantworter. Dies gilt auch dann, wenn der Arzt nur für einen Tag (z. B. Brückentag) nicht in der Praxis ist.

Dauert die Vertretung länger als eine Woche, so ist sie der KV anzuzeigen. Die schriftliche Anzeige sollte den Vertretungsgrund - z.B. Urlaub - und muss den Vertreter beinhalten. Einige KVen stellen Formulare bereit oder bieten an, die Abwesenheit direkt im KV-OnlinePortal zu hinterlegen.

Innerhalb eines Zeitfensters von 12 Monaten darf sich der Vertragsarzt bis zu einer Dauer von insgesamt 3 Monaten vertreten lassen. Hierbei sind nicht die Tage zu addieren, an denen sich der Vertragsarzt tatsächlich vertreten lässt, sondern es kommt bei durchgängiger Vertretung auf die Zeitspanne zwischen erst- und letztmaligem Vertretereinsatz an. Dauert die Abwesenheit länger als 3 Monate, so muss die Vertretung bei der KV gesondert beantragt und von ihr genehmigt werden. Vorsicht: Eine nachträgliche bzw. rückwirkende Genehmigung ist nicht möglich.

Eine regelmäßige Vertretung, z. B. einmal die Woche oder alle zwei Wochen, ist bereits ab dem ersten Tag der Vertretung gegenüber der $\mathrm{KV}$ anzeigepflichtig. Vor allem aber ist sie unzulässig, wenn die Grenze zum „Dauervertreter“ überschritten wird.

\section{Autor: Rechtsanwalt und Fachanwalt für Medizinrecht Dr. Thomas Willaschek, D+B Rechtsanwälte Partnerschaft mbB, www.db-law.de}

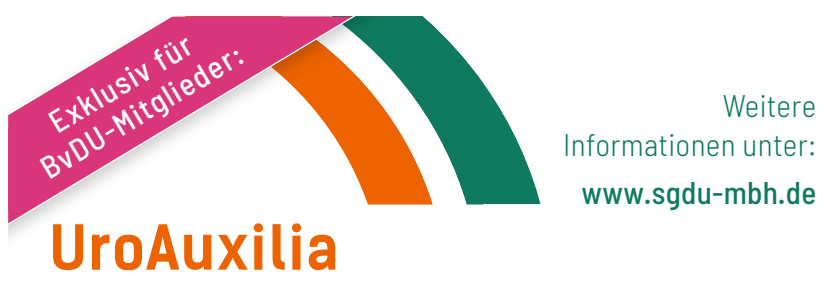

Wichtige Handreichungen für den Praxisalltag:

Folge 1: „Aktive Überwachung (Active Surveillance) beim Prostatakarzinom"

Folge 2: „Zystoskopaufbereitung in der Praxis niedergelassener Urologen"

Folge 3: „Qualitätssicherung im urologischen Labor und der Urinmikrobiologie"

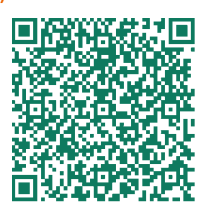




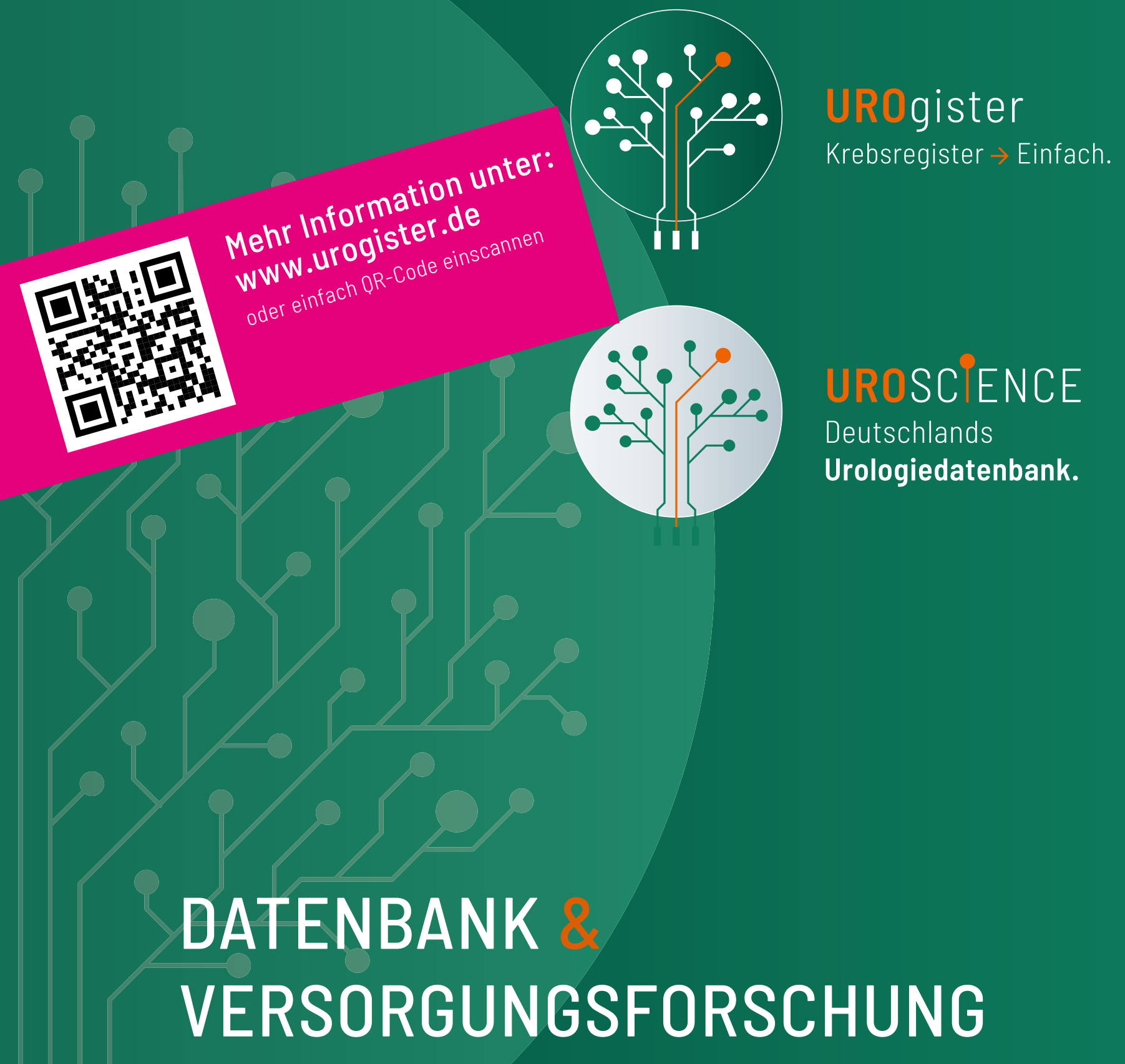

VON UROLOGEN - FÜR UROLOGEN.

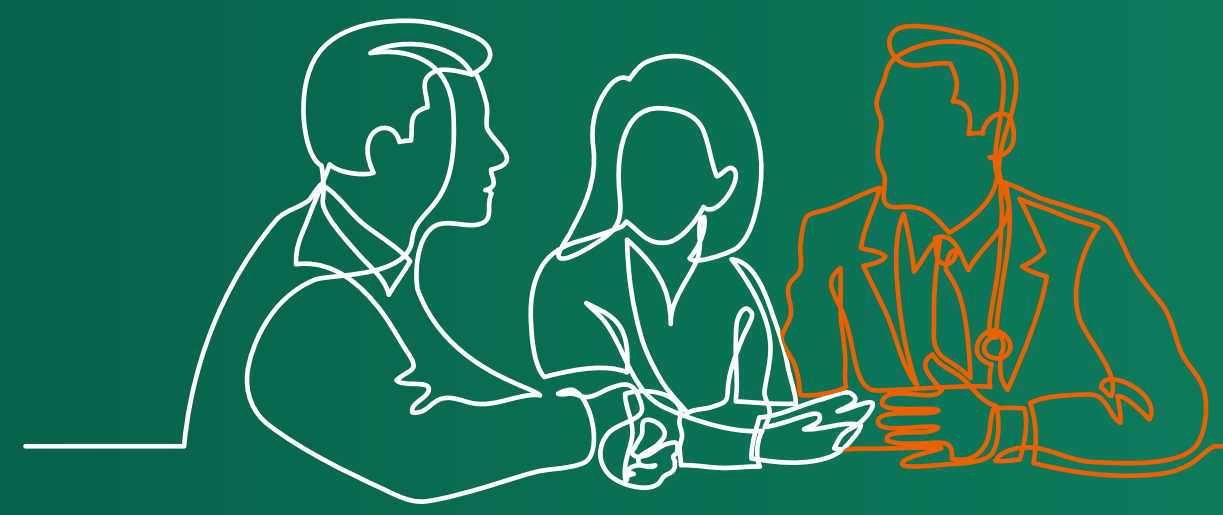




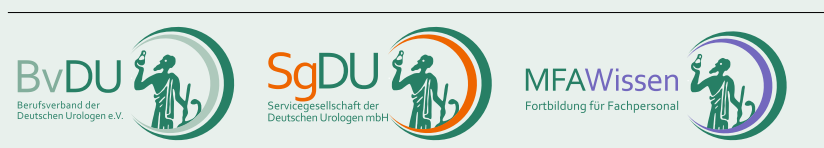

2021

12.06.2021

Mai

25.05.2021

Online

19.00-19.30 Uhr

Webinar: Update Kassenab-

rechnung | Terminservicegesetz

(TSVG) und Corona: Chancen und

Hindernisse | Schleswig-Holstein

28.-29.05.2021

Hamburg

ganztägig

12. Urologisches Sommerforum

CME-Punkte werden beantragt

Juni

01.06.2021

Online

19.00-19.30 Uhr

Update Kassenabrechnung |

Terminservicegesetz (TSVG) und

Corona: Chancen und Hindernis-

se| Sachsen

04.-05.06.2021

Berlin

ganztägig

Praxisgründungsseminar

04.-05.06.2021

Berlin

ganztägig

MFAWissen: Qualifizierungskurs

zur Hygiene und der Aufberei-

tung von Medizinprodukten

08.06.2021

Online

19.00-19.30 Uhr

Update Kassenabrechnung |

Terminservicegesetz (TSVG) und

Corona: Chancen und Hindernis-

se | Niedersachsen

09.06.2021

Online

14.30-17.30 Uhr

MFAWissen: Onkologie-Refresher

Teil 2

11.-12.06.2021

Aachen

ganztägig

MFAWissen: Qualifizierungskurs

zur Hygiene und der Aufberei-

tung von Medizinprodukten
Online

12.30-17.00 Uhr

Zertifiziertes Live-Webinar:

Grundlagentraining Transureth-

rale Therapie mit Botulinumtoxin

CME-Punkte werden beantragt

15.06.2021

Online

19.00-19.30 Uhr

Webinar: Update Kassenab-

rechnung | Terminservicegesetz

(TSVG) und Corona: Chancen und Hindernisse | Bremen

17.06.2021

Online

19.00-19.30 Uhr

Update Kassenabrechnung |

Terminservicegesetz (TSVG) und

Corona: Chancen und Hindernis-

se | Baden-Württemberg

19.-20.06.2021

Neu-Ulm

ganztägig

Praxisgründungsseminar

24.06.2021

Online

19.00-19.30 Uhr

Update Kassenabrechnung |

Terminservicegesetz (TSVG) und

Corona: Chancen und Hindernis-

se|Hessen

25.-26.06.2021

Essen

ganztägig

MFAWissen: Qualifizierungskurs

zur Hygiene und der Aufberei-

tung von Medizinprodukten

26.-27.06.2021

Bad-Nauheim

ganztägig

Praxisgründungsseminar

Juli

01.07.2021

Online

19.00-19.30 Uhr

Update Kassenabrechnung |

Terminservicegesetz (TSVG) und

Corona: Chancen und Hindernis-

se | Saarland

\section{Wichtige Hinweise zu unseren Veranstaltungen}

Unsere Veranstaltungen finden selbstverständlich unter

Berücksichtigung der geltenden Hygienevorschriften statt.

Im Einzelfall kann dies zu einer Begrenzung der Teilnehmerzahl

führen. Wenn möglich, bieten wir derzeit als Präsenzveranstaltung geplante Seminare digital, z. B. als Webinare, an. Zum Teil kann es aber ebenso zu Terminverschiebungen kommen. Wir informieren Sie natürlich rechtzeitig über mögliche Änderungen - vielen Dank für lhr Verständnis!

August

13.- -14.08 .2021$

Hamburg

ganztägig

MFAWissen: Qualifizierungskurs

zur Hygiene und der Aufbereitung von Medizinprodukten

September

03.-04.09.2021

Berlin

ganztägig

MFAWissen: Qualifizierungskurs zur Hygiene und der Aufberei-

tung von Medizinprodukten

16.09.2021

Stuttgart

09.00-18.00 Uhr

Zertifizierter Grundkurs Impfen

CME-Punkte werden beantragt

17.09.2021

Stuttgart

09.00-13.30 Uhr

Zertifiziertes Grundlagentraining

Transurethrale Therapie mit

Botulinumtoxin

CME-Punkte werden beantragt

November

06.11.2021

Frankfurt/Main

13.30-18.00 Uhr

Zertifiziertes Grundlagentraining

Transurethrale Therapie mit

Botulinumtoxin

CME-Punkte werden beantragt

13.11.2021

Online

10.00-15.30 Uhr

Zertifiziertes Live-Webinar: Impfgrundlagen für die urologische Praxis

CME-Punkte werden beantragt

\section{2.-13.11.2021}

Neumünster

ganztägig

Urologische Tumortherapie

Schleswig-Holstein

CME-Punkte werden beantragt
19.-20.11.2021

Stuttgart

ganztägig

6. Urologisches Herbstforum

CME-Punkte werden beantragt

27.11.2021

Online

13.30-18.00 Uhr

Zertifiziertes Live-Webinar:

Grundlagentraining Transurethrale Therapie mit Botulinumtoxin CME-Punkte werden beantragt

2022

März

04.-05. März 2022

Leipzig

ganztägig

7. Urologisches Frühjahrsforum CME-Punkte werden beantragt

Juni

10.-11.06.2022

Hamburg

ganztägig

13. Urologisches Sommerforum

CME-Punkte werden beantragt

Sie möchten eine Veranstaltung durchführen oder zertifizieren lassen? Sprechen Sie uns an, wir helfen gerne weiter.

Boris Rogowski

Tel: 03088722 55-1

E-Mail rogowski@sgdu-mbh.de, www.sgdu-mbh.de

Anmeldungen unter www.sgdumbh.de/veranstaltungen oder diesem QR-Code

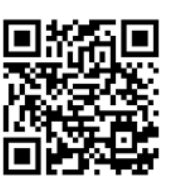

